\title{
Le curé Hébert, fondateur du Lac-Saint-Jean
}

\section{Pierre-Maurice Hébert}

Volume 55, 1988

Cent cinquante ans d'histoire religieuse au Saguenay-Lac-Saint-Jean (1838-1988)

URI : https://id.erudit.org/iderudit/1006945ar

DOI : https://doi.org/10.7202/1006945ar

Aller au sommaire du numéro

Éditeur(s)

Les Éditions Historia Ecclesiæ Catholicæ Canadensis Inc.

ISSN

0318-6172 (imprimé)

1927-7067 (numérique)

Découvrir la revue

Citer cet article

Hébert, P.-M. (1988). Le curé Hébert, fondateur du Lac-Saint-Jean. Sessions d'étude - Société canadienne d'histoire de l'Église catholique, 55, 41-47.

https://doi.org/10.7202/1006945ar

Tous droits réservés @ Les Éditions Historia Ecclesiæ Catholicæ Canadensis Inc., 1988
Ce document est protégé par la loi sur le droit d'auteur. L'utilisation des services d'Érudit (y compris la reproduction) est assujettie à sa politique d'utilisation que vous pouvez consulter en ligne.

https://apropos.erudit.org/fr/usagers/politique-dutilisation/ 


\title{
Le curé Hébert Fondateur du Lac-Saint-Jean
}

\author{
Pierre-Maurice HÉBERT, o.f.m.c. \\ Montréal, Québec
}

L'abbé Nicolas-Tolentin Hébert a toujours été considéré par les historiens et par la population comme le véritable fondateur de la région du Lac-Saint-Jean. À son jubilé d'or en 1883, la délégation du Lac-Saint-Jean le remerciait pour avoir ouvert la marche de la colonisation de la vallée du Lac-Saint-Jean'. En 1926, lors du dévoilement de son monument, à Hébertville, on proclamait unanimement le curé Hébert «père de la colonisation au Lac-Saint-Jean²». Il en fut de même aux fêtes de 1938, 1949 et 1988.

\section{Hébertville, la capitale}

Hébertville, fondée par le curé Hébert, fut la première paroisse du Lac-Saint-Jean. On détacha de son territoire Alma, Métabetchouan, etc. Elle devint aussi la première municipalité du Lac-Saint-Jean. Roberval s'en sépara en 1859. Au XIXe siècle, il y avait deux agglomérations importantes au Saguenay-Lac-Saint-Jean: Hébertville du côté du lac et Chicoutimi du côté du Saguenay.

Les deux régions ont une morphologie bien différente: les plaines autour du lac, les montagnes le long du Saguenay. C'est cette dernière région qui a été colonisée d'abord, à cause des facilités d'accès. Dix ans plus tard, en 1849, ce fut le tour du Lac-Saint-Jean, avec l'arrivée du curé Hébert. Le Saguenay garda son avance sur le Lac-Saint-Jean et s'industrialisa plus vite, grâce à ses nombreuses chutes d'eau et à ses installations portuaires en eau profonde. Le Lac-Saint-Jean, par contre, fut la première région à recevoir le chemin de fer en 1888 , directement de Québec. Il

1 Noces d'or de Messire Nicolas de Tolentin Hébert, Sainte-Anne de la Pocatière, 1884, p. 47.

2 Abbé André Laliberté, Les fêtes du Monument Hébert, Chicoutimi, 1926, p. 7, 32. 
connut alors un essor considérable. Hebertville-Station fut créée à cette occasion, entre Hébertville et Alma.

Mais donnons tout de suite quelques précisions biographiques sur le curé Hébert. Nous dégagerons ensuite certains points importants qui n'ont pu être développés dans le livre Le curé Hébert ${ }^{3}$.

\section{Notes biographiques}

Le curé Hébert était petit-fils d'Acadiens déportés qui ont fait la trame du roman Évangéline (selon Raymond Casgrain). Son père était Jean-Baptiste Hébert et sa mère Judith Lemire, de la Baie-du-Febvre. Il est né en 1810 à Saint-Grégoire, entre Bécancour et Nicolet, où les Acadiens déportés sur les côtes de l'Atlantique sont venus s'établir, dans les années 1760. Après ses études au Séminaire de Nicolet, il est ordonné prêtre à Québec en 1833 où il demeura vicaire à la paroisse mère jusqu'en 1840. Il est nommé alors curé à Saint-Pascal de Kamouraska, paroisse agricole nouvellement fondée. Il y érige l'église, le presbytère, les écoles, à l'aide de son père architecte venu vivre auprès de lui, après son emprisonnement durant les troubles de 1837-1838.

\section{L'Année 1848}

L'année 1848 est l'année clé dans l'histoire de la colonisation au Québec. Durant cette année, le grand communicateur, Bernard O'Reilly, passe comme un éclair dans le ciel québécois, avant d'entrer chez les jésuites en 1849. Cet Irlandais du Vermont, converti au catholicisme, influencé naguère par John Holmes, s'apitoyait sur le sort des Canadiens français persécutés dans les Cantons de l'Est et qui pourtant avaient aidé généreusement les Irlandais immigrants, en 1847. Il invitait les Canadiens français à s'emparer de leur sol natal afin de conserver leur nationalité. Voilà le grand mot d'ordre qui était répandu partout, à Montréal, à Québec, sur la Côte-du-Sud, etc. Le curé Hébert en a eu des échos. Les gens du Saguenay aussi. L'abbé Antoine Racine et le nouveau curé de la Malbaie, Augustin Beaudry, avec quelques laïcs, qui ouvrirent le canton de Jonquière en 1848, étaient en correspondance avec l'abbé O'Reilly4. L'abbé François Boucher et quelques autres pionniers s'apprêtaient à fonder des établissements autour du Lac-Saint-Jean en 1848, ainsi que le curé Hébert. Celui-ci réussit à y faire construire des moulins, des maisons, une église et surtout des chemins pour relier cette région lointaine au reste de la province.

3 Pierre-Maurice Hébert, Le curé Hébert, un siècle d'histoire, 1810-1888, t. I, Montréal, Éditions de l'Écho, 1988, 480 pages.

4 Journal de Québec, juin-sept. 1848. 


\section{La Côte-du-Sud}

Le curé Hébert avait derrière lui une société solide: une association de paroisses de la Côte-du-Sud composées de colons et de notables et dont le siège social était au Collège de Sainte-Anne-de-la-Pocatière. Parmi les plus actifs de ses membres se trouvait l'abbé François Pilote qui, malgré ses nombreuses tâches d'enseignant et d'administrateur au Collège, accepta volontiers le rôle de secrétaire correspondant. Il colligea différents textes pour lancer le petit livre: Le Saguenay en 1851. Ce livre aida beaucoup la cause des actionnaires et informa utilement le gouvernement et la population. Chaque actionnaire de la société fournissait $50 \$$, ce qui lui donnait droit, après cinq ans, à un lot au Lac-Saint-Jean. Avec cet argent, la société achetait les lots à $20 \$$ chacun. Il lui restait donc seulement 30 \$ pour faire les défrichements durant cinq ans, payer et nourrir les hommes (une centaine), bâtir les infrastructures d'un village, ouvrir des chemins, entre autres un chemin d'un quarantaine de milles pour atteindre le prochain village, payer l'administration, les déplacements en goélette, etc. La société n'arriva pas à faire toutes les améliorations prévues après cinq ans, ce que les actionnaires et les colons comprirent très bien. Ils acceptèrent volontiers des lots plus ou moins défrichés. L'important c'était que le village fût bâti, les chemins les plus urgents terminés, une administration en place et, de plus, qu'on puisse compter sur les secours et le soutien d'une dizaine de paroisses de la Côte-du-Sud. Le curé Hébert était toujours là pour lever des souscriptions, envoyer du linge, de la nourriture, etc., même après que les Hébertvillois eurent un curé permanent, à partir de 1857.

\section{«Emparons-nous du sol...»}

Les historiens ont beaucoup parlé des prêtres colonisateurs et des mouvements de colonisation, qui s'étendent d'ailleurs sur plus d'un siècle de notre histoire. On a parlé du besoin d'aventure, de l'appel du Grand Nord, de l'attirance de la «frontière», des joies et des bienfaits de la culture agraire, de l'agriculturisme comme d'un messianisme pour le peuple, et de l'opportunisme du clergé catholique qui aurait profité d'une belle occasion, après la déroute des troubles 1837-1838, pour affermir son pouvoir. Quoi qu'il en soit de ces idées, elles n'avaient pas cours au temps du curé Hébert. Il s'agissait plutôt, alors, de «s'emparer du sol». C'était une question de survie pour le peuple. Le règlement de la société de La Pocatière formule ainsi son objectif: «ménager à la population une entrée plus facile dans le territoire du Saguenay ${ }^{5}$ ». Ce règlement reprend d'ailleurs celui de

5 «Projet de règlement d'une association pour la Colonisation des terres du Saguenay, adopté par l'assemblée des Délégués des actionnaires, tenue à Ste-Anne, La Pocatière, jeudi le 22 mars 1849.» Archives du Collège de Sainte-Anne-de-la-Pocatière, 38-V article I; Journal de Québec, 17 juin 1849. 
Racine et Beaudry, qui parlait d'ouvrir des terres en favorisant, "par là même, le commerce ${ }^{\star}$. Il y a une parenté évidente entre les deux règlements. Le mémoire de Saint-Pascal en 1848, auquel a travaillé le curé Hébert, est plus explicite. Il se rattache au grand mouvement de colonisation qui balaie alors le Bas-Canada et il demande «les terres non encore prises situées sur les bords du lac Saint-Jean ${ }^{7}$.

\section{Origines du mouvement}

Il y aurait une étude comparative à faire des différents règlements de sociétés de colonisation en 1848 . On verrait qu'ils se rattachent à l'appel de l'abbé O'Reilly, qui lui-même se rattachait au mouvement des Irlandais et des Écossais du Haut-Canada pour la possession des terres ${ }^{8}$. Le livre de propagande de la société du curé Hébert, Le Saguenay en 1851, affiche en exergue sur sa couverture ces mots de ralliement qui retentissaient alors partout: «Emparons-nous du sol si nous voulons conserver notre nationalité». C'était en contrepartie de la prise de possession des terres par les Loyalistes et par la compagnie étrangère, la British American Land Company. Le père du curé Hébert avait toujours parlé, dans ses discours politiques, contre la mainmise étrangère qui évinçait de leur patrie les Canadiens français. L'abbé O'Reilly faisait de même. Il demeurait, en 1848 , au presbytère de Saint-Grégoire de Nicolet, d'où les nombreux cousins du curé Hébert partaient pour aller conquérir les townships ${ }^{9}$. Il s'agissait de s'emparer des terres vacantes dans la périphérie du pays, contrer ainsi l'influence étrangère et sauvegarder sa nationalité. Il y avait là un patriotisme évident.

Le clergé engourageait ce mouvement sur le plan matériel et sur le plan spirituel. Mgr Turgeon écrivait dans sa lettre circulaire du 11 août 1848: «les sommes que nous prélèverons seront employées à entourer chaque nouvel établissement, pendant son enfance, des secours religieux

6 Journal de Québec 23 septembre 1848, no. 120, p. 1. On trouve un texte non daté du «Règlement de la Société des Défricheurs du Saguenay» aux Archives du Collège de SainteAnne-de-la-Pocatière, 38-I. Un autre texte, daté du 10 juillet 1848, de la Malbaie, se trouve dans le greffe du notaire Jean Gagné qui était d'ailleurs secrétaire de la Société. Je remercie M. L'abbé Jean-Philippe Blackburn, archiviste à l'évêché de Chicoutimi pour m'avoir aidé à repérer ces documents, de même que M. l'abbé Armand Gagné de l'archevêché de Québec.

7 «Mémoire à l'appui de la requête des habitants des paroisses de St-Pascal de Kamouraska et d'autres paroisses qui désirent s'établir dans le territoire du Sauguenay.» Archives du Collège de Sainte-Anne-de-la-Pocatière, 38-IV.

8 Gilles Parent, Deux efforts de colonisation française dans les Cantons de l'Est, 1848 et 1851, Sherbrooke, Université, 1980, p. 22.

9 Léon Pouliot, S.J., Monseigneur Bourget et son temps, Montréal, Bellarmin, 1972, t. 3, 54; Journal de Québec, 14 novembre 1848: des centaines de jeunes de Saint-Grégoire de Nicolet partent pour la colonisation; les femmes sont éplorées. 
et matériels nécessaires au colon». Il proposait la formule de la Propagation de la Foi:

On se forme en décuries et centuries, comme dans l'Association de la Propagation de la Foi. Chaque paroisse a un comité local qui se compose d'un président, de deux vice-présidents, d'un secrétaire, d'un trésorier, d'un auditeur des comptes. Les officiers des comités de paroisse forment le Bureau central de Régie pour tout le comté.

Le comité central élit ses propres officiers à la majorité des suffrages, et fixe les jour et lieu de ces assemblées.

L'association de la Propagation de la Foi a, non seulement servi de modèle aux sociétés de colonisation, mais elle a aidé de ses deniers surtout à construire des églises ${ }^{10}$.

\section{Les enjeux politiques}

L'autre motif impérieux qui poussait le curé Hébert et les sociétés de colonisation à trouver de nouvelles terres était la saignée nationale que constituait le départ des Canadiens français vers les États-Unis. Des milliers partaient chaque année. De Saint-Pascal de Kamouraska, les garçons se rendaient surtout vers l'Illinois. Le seul débouché qui s'offrait alors dans le Bas-Canada, c'était la terre. Il n'y avait pas alors d'industrie au pays. L'agriculture n'était donc pas un choix, c'était une nécessité: ou bien la terre ou bien l'exil. Comme les terres des seigneuries étaient toutes prises, il fallait ouvrir de nouveaux cantons et les occuper avant qu'une mainmise étrangère ne s'en empare.

Le gouvernement, en 1848, fut aussi amené à prendre la tête du mouvement, et pas seulement le clergé. Il voulait alors devancer Louis-Joseph Papineau qui s'en servait déjà pour un retour à la politique, dans l'idée de faire abolir l'acte d'Union. Le gouvernement écarta ce dangereux politicien et investit $£ 20000$ dans la colonisation. Mais cet argent alla surtout aux Cantons de l'Est. Le Lac-Saint-Jean n'eut pas sa part proportionnellement, car on y protégeait toujours les grosses compagnies: la Hudson Bay Company, la Compagnie Price qui apportaient des revenus considérables à l'État ${ }^{11}$.

\section{Une société généreuse}

La société du curé Hébert avait été très généreuse dans ses statuts, beaucoup plus que d'autres, par exemple en allouant une rémunération au

10 Monseigneur Bourget écrivait; «Les deux associations [...] vont marcher dans la même route.» (Elgin-Grey Papers, Ottawa, Doughty, 17 juin 1848, p. 193). Voir aussi Stanislas Drapeau, Colonisation du Bas-Canada 1851-1861, Québec, 1863, pp. 222-229; Gilles Parent, Deux efforts [...], p. 34, 76; Jacques Monet, S.J., La première révolution tranquille, Montréal Fides, 1981, p. 355.

11 Léon Pouliot, S.J., Monseigneur Bourget et son temps, t. 3, p. 51. 
trésorier et au secrétaire. Elle offrait aussi un agent. Ce fut son point chanceux, car le curé Hébert assuma cette fonction gratuitement. Il a alors fait le succès de l'association, là où les autres ont échoué.

Mais l'association rencontra des difficultés particulières. D'abord à cause de la longueur du chemin à faire pour atteindre le lac Saint-Jean; de plus, des amoncellements rocheux séparaient celui-ci du reste du pays. Puis, une fois rendus dans la vallée du Lac-Saint-Jean, les colons se trouvaient très isolés. On manquait de tout: de nourriture, de vêtements, d'instruments de iravail, eic. Oni mañuquait de la préscnec familiale: ce n'est qu'à partir de 1852 que quelques femmes arrivèrent.

Le curé Hébert avait prévu ces difficultés. Avant même la fondation de l'association, il avait réuni des volontaires à Saint-Pascal de Kamouraska. Dans son mémoire envoyé au gouvernement en 1848, il spécifiait la présence nécessaire du prêtre pour le bon moral des colons au Lac-Saint-Jean. La distance était si grande, d'Hébertville aux autres établissements, qu'il fallait tout de suite une occupation massive, en groupe, pour s'entraider ${ }^{12}$. Il faudrait procurer des terres faciles à cultiver, unies et sans roche. Surtout, il ne fallait pas augmenter le prix des terres au-dessus d'un chelin, ce qui était déjà cher, considérant le manque de chemins. Il fallait apprendre à se suffire collectivement, dès la première année. Il fallait surtout beaucoup de capitaux pour engager durant cinq ans une centaine de personnes, les payer, les nourrir, etc. Ainsi les colons pauvres pourraient, non seulement gagner de l'argent, mais aussi payer leur lot en versant $10 \$$ par année avec une partie de leur salaire. Le plan du curé Hébert s'est réalisé comme prévu.

\section{Après la fondation}

Après cinq ans, lors de la dissolution de la compagnie, il resta une dette à payer, étant donné les investissements considérables. Mais cette dette fut peu à peu effacée, grâce aux bons soins du curé, à la coopération du gouvernement et du Collège de Sainte-Anne-de-la-Pocatière. Le curé Hébert avait réussi la conquête des terres du Lac-Saint-Jean. Victor Tremblay, qui a bien étudié tous les efforts de colonisation au Saguenay-Lac-Saint-Jean, a écrit.:

Parmi les nombreuses sociétés qui ont été formées, il y a un siècle, pour réaliser des entreprises de colonisation, celle des comtés de L'Islet et de Kamouraska, plus souvent appelée «Société du Curé Hébert» est apparemment celle qui fut la mieux organisée et dont l'œuvre eut le meilleur succès. C'est elle qui, dans des conditions particulièrement difficiles, entreprit, en 1849, et réalisa la conquête de la vallée du Lac-Saint-Jean ${ }^{13}$.

12 «Mémoire à l'appui de la requête [...]"

13 Rapport de l'Archiviste de la province de Québec, 1948-1949, p. 277. 
Les débuts furent difficiles pour les premiers colons au Lac-SaintJean, qui furent aussi héroïques que leur chef. On peut dire cependant que, grâce à la sollicitude constante du curé, ce fut moins difficile au Lac-SaintJean qu'ailleurs. On connaît des situations beaucoup plus pénibles de plusieurs colons isolés dans les Cantons de l'Est ${ }^{14}$.

En 1870, le feu ravagea, comme on le sait, la région du Lac-SaintJean et du Saguenay. Heureusement, Hébertville fut en partie épargnée avec Chicoutimi. Des secours s'organisèrent rapidement. Hébertville resta l'endroit du Lac-Saint-Jean où on venait de partout pour les services sociaux: justice, soins médicaux, magasins, etc. Le curé Hébert, alors promu à Saint-Louis de Kamouraska, était toujours en correspondance avec ses «amis de la colonisation» du Lac-Saint-Jean. Mais il était aussi très actif sur la Côte-du-Sud, non seulement pour Kamouraska, centre de district judiciaire, mais aussi pour tous les mouvements socio-culturels et religieux qui avaient souvent leur centre au collège de La Pocatière. Nicolas-Tolentin Hébert est décédé dans son presbytère en 1888 , toujours curé de Kamouraska.

14 «des familles, en assez grand nombre, passent des semaines et des mois entiers sans voir aucun pain, vivant d'herbes et de racines sauvages. Leurs vêtemens et leurs ameublements vont de pair avec leur nourriture. Une chétive hutte de bois rond, couverte seulement de tuiles de cèdre et surmontée d'une cheminée en terre, voilà l'habitation de la majeure partie». Pierre-Jacques Bédard, prêtre, cité par Gilles Parent, Deux efforts [...], p. 24. 